\title{
Combined perioperative plasma endoglin and VEGF-A assessment in colorectal cancer patients
}

\author{
Piotr Myśliwiec¹, Krystyna Pawlak², Adam Kukliński', Bogusław Kędra1 \\ $12^{\text {nd }}$ Department of General and Gastroenterological Surgery and \\ ${ }^{2}$ Department of Nephrology and Transplantology, Medical University of Bialystok, Poland
}

\begin{abstract}
Colorectal cancer growth and spread is absolutely dependent on angiogenesis with vascular endothelial growth factor (VEGF) being the most important cytokine involved in the process. Endoglin, a membrane co-receptor for TGF- $\beta$, has recently emerged as a sensitive index of cancer stage. There is now sufficient evidence indicating that microvessel density assessed by endoglin-immunostaining correlates with stage of colorectal cancer and patient survival. An association of a soluble form of endoglin with lymph node and distant metastases has recently been reported in two studies. Both of them used local elaborated immunoassays for endoglin assessment. The aim of our study was to determine the efficacy of plasma endoglin, assessed using a commercial kit, as a marker of tumor spread and distant metastases in colorectal cancer patients. We studied 48 colorectal cancer patients, compared with 22 healthy subjects, using ELISA. We observed that colorectal cancer patients had increased plasma VEGF-A, but not endoglin levels. However, we found an association of plasma endoglin with the stage of malignancy. Endoglin levels were increased in metastasis-positive patients when compared to both metastasis-negative patients and healthy volunteers. Plasma endoglin correlated with VEGF-A, CEA and CA19.9. Endoglin assessment in plasma does not seem useful as a maker of colorectal cancer. Our observations indicate however that it might be helpful in selecting patients with metastatic disease.
\end{abstract}

Key words: colorectal cancer, endoglin, VEGF-A, angiogenesis

\section{Introduction}

Colorectal cancer is one of the leading cancers in the western countries with half a million deaths worldwide every year [1]. At diagnosis almost a third of all patients already have metastatic disease and nearly $50 \%$ of all diagnosed colorectal cancer patients will subsequently develop metastases with often fatal prognosis [2]. Molecular markers of solid tumors can not only be useful in cancer classification but they can also predict dissemination of the disease [3]. So far, no perfect molecular marker has been found to predict disease progression.

Tumor growth and spread are absolutely dependent on their ability to induce angiogenesis [4] with vascular endothelial growth factor (VEGF) as the most important cytokine involved in the process [5]. VEGF plays an important role in progression, invasion and spread of colorectal cancer by influencing proliferation and migration of endothelial cells [6]. It has also been

Correspondence: P. Mysliwiec, $2^{\text {nd }}$ Dept. of General and Gastroenterogical Surgery, Medical University of Bialystok, M. Skłodowskiej-Curie 24a, 15-276 Białystok, Poland; e-mail: mpiotr@umwb.edu.pl reported to be a useful marker of prognosis by significantly correlating with angiolymphatic invasion, lymph node status and depth of invasion, although it was not an independent prognostic factor [7].

Total VEGF is not a sufficient diagnostic tool in patients with colorectal cancer because of poor sensitivity $(36 \%)$ [8]. The ligands of the VEGF family include VEGF-A, VEGF-B, VEGF-C, VEGF-D and VEGF-E [9]. VEGF-A is the most abundantly expressed in colorectal cancer tissues and therefore seems to be of greater value then total VEGF [5].

One of the recently emerging cytokines associated with angiogenesis is endoglin [10]. Endoglin, also known as CD105, is a trans-membrane co-receptor for TGF- $\beta 1$ and TGF- $\beta 3$. It modulates the cell response to those mediators. Biochemically it is a $180 \mathrm{kDa}$ glycoprotein. Beside angiogenesis it plays a role in hematopoiesis and cardiovascular system development [11]. CD105 is induced by hypoxia. As it is present mainly in new vessels, endoglin proved to be very useful in assessment of newly formed vessels in malignant neoplasms [12]. Antibodies raised against endoglin were also used for tumor imaging $[10,13]$. Intra-tumor microvessel density determined by immunohistochem- 
ical staining for endoglin has been reported to be an indicator of poor prognosis in many types of solid neoplasia such as: breast carcinoma, cervical cancer, endometrial carcinoma, gastric carcinoma, melanoma, some testicular tumors, non-small cell lung cancer, prostate cancer, renal cell carcinoma and squamous cell carcinoma [14].

Many reports indicate that endoglin assessed immunohistochemically in colorectal cancer correlates not only with tumor microvessel density, but also with survival $[7,15]$. It has been also reported as a valuable parameter predicting patients having an increased risk of developing metastatic disease [16]. It correlates with tissue expression of VEGF [17].

Endoglin is not only expressed on cell surface since its soluble form can also be detected in blood. Soluble endoglin was shown to be increased in many tumors including: myeloid malignancies and breast cancer [18]. Two studies also documented elevated levels of circulating endoglin in colorectal cancer patients $[15,19]$. Both used local immunoassays for its detection.

The aim of our study was to assess the efficacy of plasma endoglin when compared with VEGF-A, as a marker of tumor spread and distant metastases in colorectal cancer patients.

\section{Materials and methods}

Tissue samples. This study was performed on plasma samples from 48 patients with colorectal cancer treated in the II Department of General and Gastroenterological Surgery, Medical University of Bialystok. Control group consisted of 22 healthy subjects.

Among the colorectal cancer patients there were 25 men and 23 women (Table 1). The mean age was 65.2 (32 to 83). The cancer was localized in rectum in 27 patients, in sigmoid colon in 8 , in cecum in 5 , in ascending colon in 4 , in descending colon in 1 and in transverse colon in 3 patients. 29 patients underwent curative resection and 19 patients - palliative resection. In all cases pathologic examination indicated adenocarcinoma. According to Dukes' classification, 6 patients were at stage A, 12 at stage B, 16 at stage $\mathrm{C}$ and 14 at stage D. In all colorectal cancer patients blood samples were collected before surgery and at post-operative days 3 and 10 .

The control group comprised 11 men and 11 women, aged 32 to 78 (mean 64.9). They were healthy subjects. Patients and volunteers with clinical or laboratory signs of infection were excluded from the study. Exclusion criteria for control subjects also comprised malignancy, liver and kidney diseases, chronic connective tissue and skin diseases. Ethics committee approval and informed consent from all patients were obtained.

Blood samples from patients with colorectal cancer were obtained prior to any treatment. After an overnight fast, $4.5 \mathrm{ml}$ of venous blood were poured into a tube containing $0.5 \mathrm{ml}$ EDTA. Immediately after sampling, specimens were centrifuged at $1000 \mathrm{rpm}$ for 15 minutes in $4^{\circ} \mathrm{C}$. The supernatant - platelet poor plasma was transferred to Eppendorf's tube and frozen at $-70^{\circ} \mathrm{C}$ until analysis.

VEGF-A was determined according to George et al. [20] and endoglin - using a commercially available ELISA kit purchased from Research Diagnostics Inc., USA.

Statistical analysis. Data were analyzed using Statistica 5.1. computer software (StatSoft Inc, Tulsa). Normality of variable distribution was tested using Shapiro-Wilk W test. The normally distributed data were expressed as mean \pm SEM. The non-Gaussian
Table 1. Clinical and pathological characteristics of the colorectal cancer patients.

\begin{tabular}{|c|c|c|}
\hline & Patient number & $(\%)$ \\
\hline \multicolumn{3}{|l|}{ Gender: } \\
\hline Male & 25 & $(52.1)$ \\
\hline Female & 23 & $(47.9)$ \\
\hline \multicolumn{3}{|l|}{ Tumor site: } \\
\hline Right colon & 9 & $(18.8)$ \\
\hline I eft colon & 4 & $(8.3)$ \\
\hline Sigmoid colon & 8 & $(16.7)$ \\
\hline Rectum & 27 & $(56.2)$ \\
\hline \multicolumn{3}{|l|}{ Resection type: } \\
\hline Curative & 29 & $(60.4)$ \\
\hline Palliative & 19 & $(39.6)$ \\
\hline \multicolumn{3}{|c|}{ Dukes' classification } \\
\hline Stage A & 6 & $(12.5)$ \\
\hline Stage B & 12 & $(25.0)$ \\
\hline Stage C & 16 & $(33.3)$ \\
\hline Stage D & 14 & $(29.2)$ \\
\hline \multicolumn{3}{|l|}{ Tumor infiltration: } \\
\hline pll & 6 & $(12.5)$ \\
\hline pT2 & 9 & (18.8) \\
\hline pT3 & 27 & $(56.2)$ \\
\hline pT4 & 6 & $(12.5)$ \\
\hline \multicolumn{3}{|l|}{ Lymph node status: } \\
\hline pNO & 18 & $(37.5)$ \\
\hline $\mathrm{pNl}$ & 10 & $(20.8)$ \\
\hline $\mathrm{pN} 2$ & 20 & $(41.7)$ \\
\hline \multicolumn{3}{|l|}{ Metastases status: } \\
\hline $\mathrm{pM} 0$ & 34 & $(70.8)$ \\
\hline pM1 & 14 & $(29.2)$ \\
\hline \multicolumn{3}{|c|}{ Grade of differentiation: } \\
\hline G2 & 29 & $(60.4)$ \\
\hline G3 & 19 & $(39.6)$ \\
\hline
\end{tabular}

data were presented as median (quartile range). Non-normally distributed variables were log-transformed prior to regression analysis. ANOVA was employed to test differences between groups. The Mann-Whitney test or Student's t test were used in statistical analysis to compare differences between individual groups. For differences between assessments performed on days 0,3 or 10 dependent t-Student test or Wilcoxon test were used. Correlations were determined using Spearman's analysis. A two-tailed $p$ value $<0.05$ was considered to be statistically significant.

\section{Results}

We found significant increases in VEGF-A concentrations in plasma of patients with colorectal cancer in 


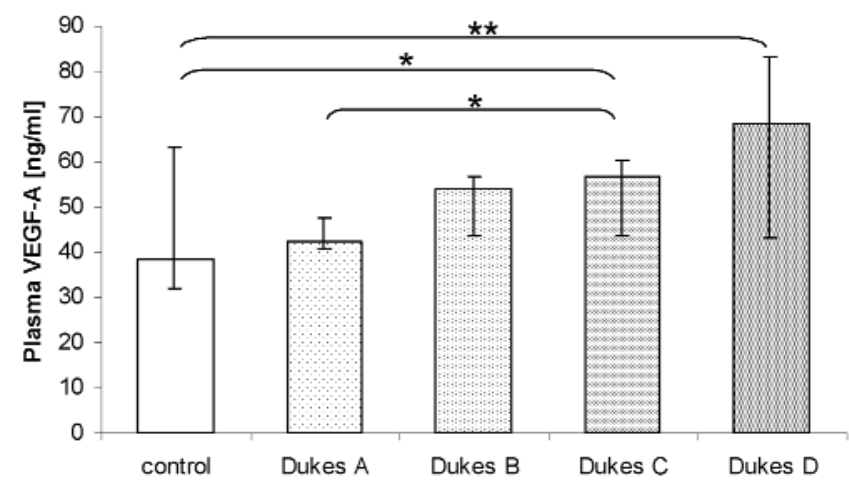

Fig. 1. Preoperative concentration of VEGF-A in plasma of normal controls (control) and patients with colorectal cancer at stages A$\mathrm{D}$ according to Dukes. (Results are given as median \pm quartile range. Significant differences of Mann-Whitney U test are marked with $*_{\text {- }}$ $<0.05$ or $* *_{\text {- }}$ p $<0.01$.

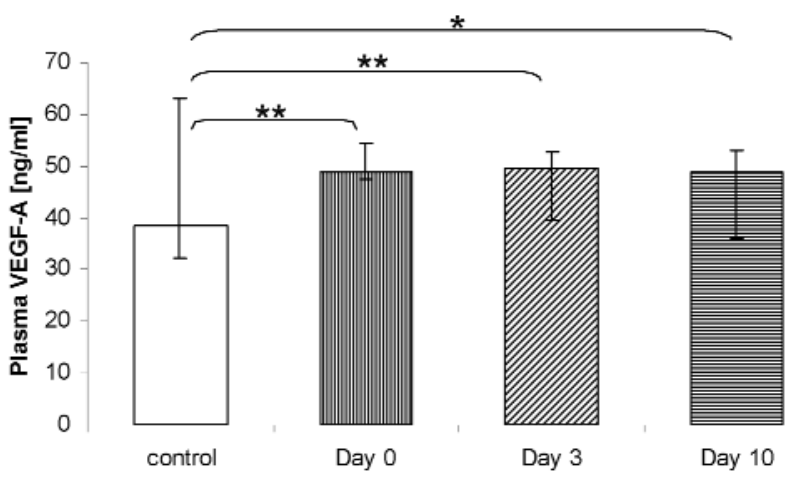

Fig. 3. Influence of operation on the concentration of VEGF-A in plasma of patients with colorectal cancer. For comparison the control values are also shown (control). (Results are given as median $+/$ - quartile range. Differences significant according to MannWhitney $U$ test are marked as: $*-p<0.05 ; * *-p<0.01$. No significant differences between days 0,3 or 10 were found).

Dukes' stage $\mathrm{C}(\mathrm{p}<0.05)$ and $\mathrm{D}(\mathrm{p}<0.01)$ (Fig. 1), when compared to the control group. Mean levels of plasma VEGF-A in all colorectal cancer patients combined were also greater than in healthy subjects $(p<0.01)$ (Fig. 3). Operation did not change VEGF-A level significantly in colorectal cancer patients (Fig. 3). The levels of VEGF-A postoperatively were still greater in cancer groups than in the control group both on day 3 $(p<0.01)$ and $10(p<0.05)$, with no significant difference between the days.

Endoglin was detectable in plasma of $91.7 \%$ of the operated patients and in $86.4 \%$ of the normal controls. The levels of endoglin obtained with the commercial ELISA kit were greater than those reported by Li [16], who used an indirect sandwich ELISA kit. At the same time they were about 8-fold lower than those reported in serum [31]. In contrast to VEGF-A, no significant difference in plasma endoglin was found preoperatively between colorectal cancer patients and healthy volunteers (Fig. 4).

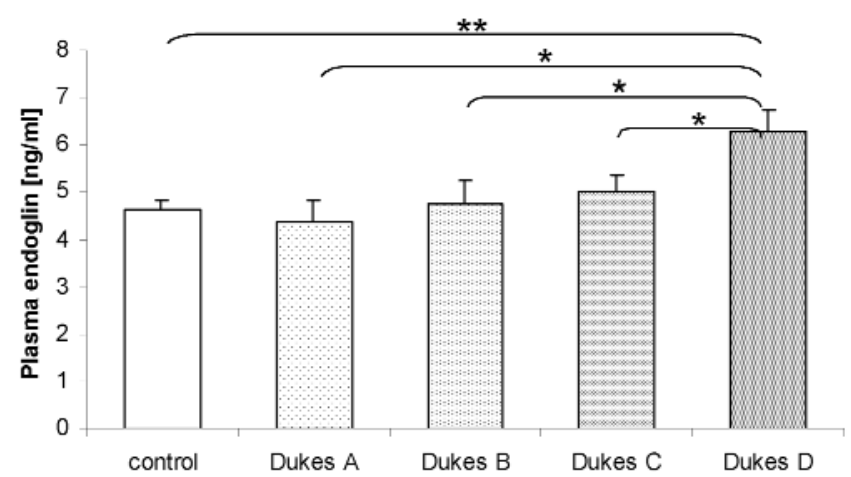

Fig. 2. Preoperative concentration of endoglin in plasma of normal controls (control) and patients with colorectal cancer at stages A$\mathrm{D}$ according to Dukes. (Results are given as mean \pm SEM. Significant differences are marked with $*_{-} \mathrm{p}<0.05$ or $*^{*}$ - $\mathrm{p}<0.01$.

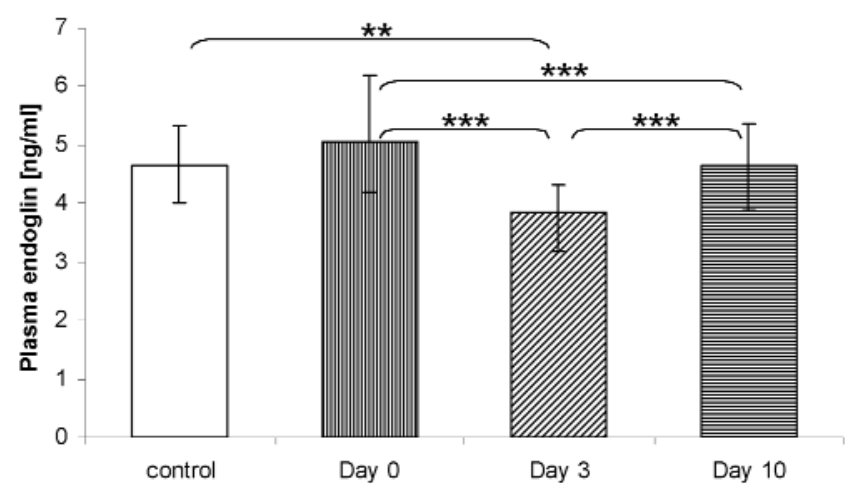

Fig. 4. Influence of operation on the concentration of endoglin in plasma of patients with colorectal cancer. For comparison the control values are also shown (control). (Results are given as median + - quartile range. Differences significant according to MannWhitney U test are marked as: $\left.{ }^{* *} *_{-} \mathrm{p}<0.01 ; * * *_{-} \mathrm{p}<0.001\right)$

When analyzing disease staging according to Dukes, there was an apparent gradual rise in endoglin level from groups $\mathrm{A}$ to $\mathrm{D}$ with a significant difference between group $\mathrm{D}$ and healthy subjects ( $\mathrm{p}<0.01$ ) (Fig. 2). Endoglin levels in the group $\mathrm{D}$ were greater than in groups A, B or $\mathrm{C}(\mathrm{p}<0.05)$. This was also reflected in the significantly greater value of endoglin in metastasis-positive (Dukes D) compared with metastasis-negative patients (Dukes $\mathrm{A}, \mathrm{B}$ and $\mathrm{C}$ combined) $(\mathrm{p}<0.01)$ (data not shown).

As shown in Fig. 6, after the operation endoglin levels initially (on postoperative day 3 ) decreased substantially when compared with preoperative concentrations $(p<0.001)$, then they rose at postoperative day 10 to reach greater values than on postoperative day 3 $(\mathrm{p}<0.001)$. They remained lower than preoperatively $(p<0.001)$. The endoglin concentrations, assessed postoperatively at day 3 were significantly lower than those in healthy subjects $(\mathrm{p}<0.01)$.

When analyzing relationship between laboratory and clinical parameters of colorectal cancer patients, 
Table 2. Chosen correlations between pathological or laboratory parameters and concentrations of VEGF-A and endoglin in patients with colorectal cancer.

\begin{tabular}{|l|c|c|}
\hline \multicolumn{1}{|c|}{ Correlation between } & Spearman's R & $\mathrm{p}$ \\
\hline VEGF-A and endoglin concentrations in plasma preoperatively & 0.43 & $<0.05$ \\
\hline VEGF-A and endoglin concentrations on post-operative day 3 & 0.51 & $<0.05$ \\
\hline VEGF-A and endoglin concentrations on post-operative day 10 & 0.58 & $<0.05$ \\
\hline Dukes' stage and plasma endoglin before operation & 0.32 & $<0.05$ \\
\hline Dukes' stage and plasma endoglin on post-operative day 3 & 0.52 & $<0.05$ \\
\hline Dukes' stage and plasma endoglin on post-operative day 10 & 0.42 & $<0.05$ \\
\hline CEA in serum and plasma endoglin preoperatively & 0.40 & $<0.05$ \\
\hline CA19.9 in serum and plasma endoglin preoperatively & 0.39 & $<0.05$ \\
\hline CA19.9 in serum and plasma VLGF-A postoperatively & 0.49 & $<0.05$ \\
\hline
\end{tabular}

we found a strong positive correlation between VEGFA levels, as assessed on day 10, and postoperative CA 19-9 (Table 2). Incidence of distant metastases was significantly greater with increased VEGF-A as assessed postoperatively both on day 3 and day 10 , as we demonstrated using Mann-Whitney $\mathrm{U}$ test $(\mathrm{p}<0.05)$ (data not shown). However, the preoperative VEGF-A values showed no significant difference between metastases-negative and -positive patients. Using the Spearman's test we found a moderate positive correlation $(\mathrm{R}=0.43)$ between plasma concentration of VEGF-A and endoglin in patients with colorectal cancer $(p<0.05)$.

Endoglin concentrations in plasma correlated positively with several indices of colorectal cancer stage. We found a moderate association between endoglin concentrations in plasma and Dukes stage, independently of the day of endoglin assessment (Table 2). Similarly, there was a correlation between endoglin and CEA as well as CA19.9 level in serum.

\section{Discussion}

We present for the first time results of plasma endoglin assessment in colorectal cancer patients using commercially available ELISA test. Although endoglin levels were not greater in colorectal cancer patients than in healthy control, they were strongly associated with distant metastases. In patients with metastases (stage D according to Dukes) endoglin had markedly elevated values not only when compared to the control group, but also to all other subgroups of colorectal cancer patients (Fig. 2).

Angiogenesis is a key process in colorectal cancer growth, invasion and dissemination. Among numerous cytokines involved in new vessel formation, VEGF has been among the best characterized [21]. Of the whole VEGF family, VEGF-A seems to be the most important for colorectal cancer spread [22]. Indeed, VEGF-A expression enhancement has been previously demonstrated in colorectal cancer patients as compared to normal controls. An association of VEGF-A tissue expression with tumour grade and size was also reported [5]. It was also shown that VEGF-A mRNA expression in colorectal mucosa strongly correlates with liver metastases and that VEGF-A concentrations in serum are an independent predicting factor for survival in patients with colorectal cancer [23].

Our results confirm a significant correlation between VEGF-A and clinical stages of colorectal cancer. We demonstrated increases in plasma cytokine levels of patients when compared with healthy subjects. We also found a strong positive association of VEGF-A plasma concentrations assessed postoperatively and the presence of distant metastases. This was in line with postoperative CA19.9 values. We observed a moderate unsignificant increase in VEGF-A plasma levels following the operation performed in colorectal cancer patients. This might be the result of thrombocytes and neutrophils breakdowns, containing substantial amounts of VEGF, as a result of surgical manipulations [24].

Endoglin, a hypoxia-induced membrane glycoprotein [25], is part of the TGF- $\beta$ receptor complex; it can modulate cellular responses to TGF- $\beta$ implicated in the early steps of angiogenesis [26]. Endoglin modulates endothelial-mesenchymal signaling and enhances cell proliferation [14]. Normal levels of endoglin are mandatory for physiologic angiogenic activity [27]. Endoglin knockout mice died of defective vascular development during early gestation [28]. Endoglin gene mutations are associated with hereditary hemorrhagic teleangiectasia type 1 , characterized by arteriovenous malformations and bleedings [14].

It has been shown that endoglin expression correlates with tumor infiltration, occurrence of metastases 
and nodal involvement, but not with tumor site or degree of differentiation. The number of endoglin-positive vessels was significantly associated with a decreased rate of overall survival [16].

In contrast to VEGF-A, we did not find any significant difference in plasma endoglin between colorectal cancer patients, as assessed preoperatively, and healthy volunteers (Fig. 4). This is in line with findings by Takahashi et al. [19]. At the same time we observed association of plasma endoglin with stage of malignancy. We demonstrated an apparent gradual rise in endoglin with advancing Dukes' stages. Our results confirm the increased level of endoglin in plasma of patients with metastatic colorectal cancer (Figs. 2, 3). Endoglin was also associated with other parameters such as: CEA and CA19.9 in serum (Table 2). Thus endoglin seems to be a valuable marker of colorectal cancer invasion and spread.

Soluble endoglin has been reported to rise in plasma of breast cancer patients who developed metastases as compared to both normal controls and cancer patients without distant spread [29]. Elevated serum endoglin was associated with distant metastases not only in breast, but also in colorectal cancer and in other solid tumors [19]. Also Li et al. [15] using an indirect sandwich ELISA described a correlation between plasma endoglin and Dukes' stages.

Surgical treatment of colorectal cancer resulted in decreased endoglin concentrations as assessed at day 3 and then their rise at day 10 (Fig. 4). The postoperative endoglin levels still correlated with clinical stage. The explanation of endoglin decrease after the operation in colorectal cancer patients might be at least in part due to pro-inflammatory cytokines, such as tumour necrosis factor $\alpha$ action. TNF- $\alpha$ has been reported to downregulate endoglin at post-transcriptional level [15]. Li et al. [15] did not find significant difference between pre- and postoperative samples from 14 colorectal cancer patients, suggesting that postoperative angiogenesis during the process of wound healing is likely to overshadow a lowered endoglin level following removal of the tumour.

We have found a significant correlation between plasma VEGF-A and endoglin in colorectal cancer patients $(\mathrm{R}=0.043, \mathrm{p}<0.05)$. So far, VEGF-A has been regarded as one of the most important angiogenic cytokines in colorectal cancer. However, based on our results, it was endoglin that showed stronger association with distant metastases. We suggest that endoglin might be a better marker for assessing cancer stage and especially in selecting patients at risk of developing metastatic disease.

Does endoglin offer anything more than a diagnostic tool for colorectal cancer patients? In metastatic colorectal cancer antiangiogenesis drugs have been claimed a promising modality of treatment [30]. Anti-
VEGF-A humanized mouse monoclonal antibody (bevacizumab) in addition to chemotherapy has been reported to improve survival of patients with previously untreated metastatic colorectal cancer [31]. However, bevacizumab used in combination with Irinotecan, fluorouracil or leucovorin for the treatment of metastatic colorectal cancer has many side effects. The common ones include hypertension, proteinuria, bleeding and impaired wound healing. Infrequently life-threatening complications have been reported, such as arterial thrombosis, gastrointestinal perforation and reversible focal posterior leukoencephalopathy [32].

Endoglin as a cell-surface glycoprotein of newly formed microvessels is another promising target for antiangiogenic therapy, especially in cancer patients at risk of developing metastases [12]. Different in vitro and in vivo studies have demonstrated that antiendoglin monoclonal antibodies induced long-lasting suppression of tumor growth and metastasis in different human and murine tumor models [13]. Preliminary experimental data confirmed high efficacy of antiendoglin treatment without severe toxicity [33]. A monoclonal antibody against endoglin linked to ricinA is presently in preclinical phase [34].

To summarize, we showed that endoglin assessment in plasma does not seem useful as a maker of colorectal cancer. Our observations indicate, however, that it might be helpful in selecting patients with metastatic disease. We suggest that patients with high values of endoglin, as well as VEGF-A should be scanned for metastases.

\section{References}

[1] Saunders M, Iveson T. Management of advanced colorectal cancer: state of the art. Br J Cancer. 2006;95:131-138.

[ 2] Hurwitz H, Kabbinavar F. Bevacizumab combined with standard fluoropyrimidine-based chemotherapy regimens to treat colorectal cancer. Oncology. 2005;69 Suppl 3:17-24.

[3] Tebbutt NC, Cattell E, Midgley R, Cunningham D, Kerr D. Systemic treatment of colorectal cancer. Eur J Cancer. 2002; 38:1000-1015.

[4] Folkman J. Tumor angiogenesis and tissue factor. Nat Med. 1996;2:167-168.

[5] Hanrahan V, Currie M, Gunningham S, Morrin H, Scott P, Robinson $\mathrm{B}$ et al. The angiogenic switch for vascular endothelial growth factor (VEGF)-A, VEGF-B, VEGF-C, and VEGF-D in the adenoma-carcinoma sequence during colorectal cancer progression. J Pathol. 2003;200:183-194.

[6] Stoeltzing O, Liu W, Reinmuth N, Parikh A, Ahmad SA, Jung YD et al. Angiogenesis and antiangiogenic therapy of colon cancer liver metastasis. Ann Surg Oncol. 2003;10:722-733.

[ 7] Saad RS, Liu YL, Nathan G, Celebrezze J, Medich D, Silverman JF. Endoglin (CD105) and vascular endothelial growth factor as prognostic markers in colorectal cancer. Mod Pathol. 2004;17:197-203.

[ 8] Broll R, Erdmann H, Duchrow M, Oevermann E, Schwandner O, Markert $\mathrm{U}$ et al. Vascular endothelial growth factor (VEGF)--a valuable serum tumour marker in patients with colorectal cancer? Eur J Surg Oncol. 2001;27:37-42. 
[ 9] Sun XF, Zhang H. Clinicopathological significance of stromal variables: angiogenesis, lymphangiogenesis, inflammatory infiltration, MMP and PINCH in colorectal carcinomas. Mol Cancer. 2006;5:43.

[10] Akagi K, Ikeda Y, Sumiyoshi Y, Kimura Y, Kinoshita J, Miyazaki $\mathrm{M}$ et al. Estimation of angiogenesis with antiCD105 immunostaining in the process of colorectal cancer development. Surgery. 2002;131:S109-113.

[11] Cheifetz S, Bellon T, Cales C, Vera S, Bernabeu C, Massague $\mathrm{J}$ et al. Endoglin is a component of the transforming growth factor-beta receptor system in human endothelial cells. $J$ Biol Chem. 1992;267:19027-19030.

[12] Duff SE, Li C, Garland JM, Kumar S. CD105 is important for angiogenesis: evidence and potential applications. Faseb $J$. 2003; 17:984-992.

[13] Maio M, Altomonte M, Fonsatti E. Is it the primetime for endoglin (CD105) in the clinical setting? Cardiovasc Res. 2006;69:781-783.

[14] Fonsatti E, Maio M. Highlights on endoglin (CD105): from basic findings towards clinical applications in human cancer. J Transl Med. 2004;2:18.

[15] Li C, Gardy R, Seon BK, Duff SE, Abdalla S, Renehan A et al. Both high intratumoral microvessel density determined using CD105 antibody and elevated plasma levels of CD105 in colorectal cancer patients correlate with poor prognosis. $\mathrm{Br}$ $J$ Cancer. 2003; 88:1424-1431.

[16] Romani AA, Borghetti AF, Del Rio P, Sianesi M, Soliani P. The risk of developing metastatic disease in colorectal cancer is related to CD105-positive vessel count. J Surg Oncol. 2006;93:446-455.

[17] Yu JX, Zhang XT, Liao YQ, Zhang QY, Chen H, Lin M et al. Relationship between expression of CD105 and growth factors in malignant tumors of gastrointestinal tract and its significance. World J Gastroenterol. 2003;9:2866-2869.

[18] Calabro L, Fonsatti E, Bellomo G, Alonci A, Colizzi F, Sigalotti L et al. Differential levels of soluble endoglin (CD105) in myeloid malignancies. $J$ Cell Physiol. 2003;194:171-175.

[19] Takahashi N, Kawanishi-Tabata R, Haba A, Tabata M, Haruta $\mathrm{Y}$, Tsai $\mathrm{H}$ et al. Association of serum endoglin with metastasis in patients with colorectal, breast, and other solid tumors, and suppressive effect of chemotherapy on the serum endoglin. Clin Cancer Res. 2001;7:524-532.

[20] George ML, Dzik-Jurasz AS, Padhani AR, Brown G, Tait DM, Eccles SA et al. Non-invasive methods of assessing angiogenesis and their value in predicting response to treatment in colorectal cancer. Br J Surg. 2001;88:1628-1636.

[21] Messersmith W, Laheru D, Hidalgo M. Recent advances in the pharmacological treatment of colorectal cancer. Expert Opin Investig Drugs. 2003;12:423-434.
[22] George ML, Tutton MG, Janssen F, Arnaout A, Abulafi AM, Eccles SA et al. VEGF-A, VEGF-C, and VEGF-D in colorectal cancer progression. Neoplasia. 2001;3:420-427.

[23] Chin KF, Greenman J, Reusch P, Gardiner E, Marme D, Monson JR. Vascular endothelial growth factor and soluble Tie-2 receptor in colorectal cancer: associations with disease recurrence. Eur J Surg Oncol. 2003;29:497-505.

[24] Werther K, Christensen IJ, Nielsen HJ. Determination of vascular endothelial growth factor (VEGF) in circulating blood: significance of VEGF in various leucocytes and platelets. Scand J Clin Lab Invest. 2002;62:343-350.

[25] Sanchez-Elsner T, Botella LM, Velasco B, Langa C, Bernabeu C. Endoglin expression is regulated by transcriptional cooperation between the hypoxia and transforming growth factorbeta pathways. J Biol Chem. 2002;277:43799-43808.

[26] Li C, Hampson IN, Hampson L, Kumar P, Bernabeu C, Kumar S. CD105 antagonizes the inhibitory signaling of transforming growth factor beta1 on human vascular endothelial cells. The Faseb Journal: Official Publication of the Federation of American Societies For Experimental Biology. 2000;14:55-64.

[27] Jerkic M, Rodriguez-Barbero A, Prieto M, Toporsian M, Pericacho M, Rivas-Elena JV et al. Reduced angiogenic responses in adult Endoglin heterozygous mice. Cardiovasc Res. 2006;69:845-854

[28] Li DY, Sorensen LK, Brooke BS, Urness LD, Davis EC, Taylor DG et al. Defective angiogenesis in mice lacking endoglin. Science. 1999;284:1534-1537.

[29] Li C, Guo B, Wilson P, Stewart A, Byrne G, Bundred N et al. Plasma levels of soluble CD105 correlate with metastasis in patients with breast cancer. Int J Cancer. 2000;89:122-126.

[30] McCarthy M. Antiangiogenesis drug promising for metastatic colorectal cancer. Lancet. 2003;361:1959.

[31] Hurwitz H, Fehrenbacher L, Novotny W, Cartwright T, Hainsworth J, Heim W et al. Bevacizumab plus irinotecan, fluorouracil, and leucovorin for metastatic colorectal cancer. N Engl J Med. 2004;350:2335-2342.

[32] Maharaj AS, D'Amore PA. Roles for VEGF in the adult. Microvasc Res. 2007;74:100-113.

[33] Seon BK, Takahashi N, Haba A, Matsuno F, Haruta Y, She $\mathrm{XW}$ et al. Angiogenesis and metastasis marker of human tumors. Rinsho Byori. the Japanese Journal of Clinical Pathology. 2001;49:1005-1013.

[34] Dome B, Hendrix MJ, Paku S, Tovari J, Timar J. Alternative vascularization mechanisms in cancer: Pathology and therapeutic implications. Am J Pathol. 2007;170:1-15.

Submitted: 26 August, 2008 Accepted after reviews: 26 October, 2008 